CLNS 95/1355

ISAS/EP/95/93

\title{
On Ising Correlation Functions with Boundary Magnetic Field
}

\author{
Robert Konik and André LeClair \\ Newman Laboratory \\ Cornell University \\ Ithaca, NY 14853 \\ and \\ Giuseppe Mussardo \\ International School for Advanced Studies and INFN \\ 34014 Trieste
}

\begin{abstract}
Exact expressions of the boundary state and the form factors of the Ising model are used to derive differential equations for the one-point functions of the energy and magnetization operators of the model in the presence of a boundary magnetic field. We also obtain explicit formulas for the massless limit of the one-point and two-point functions of the energy operator.
\end{abstract}

$8 / 95$ 


\section{Introduction}

Quantum Field Theories with boundary conditions [1] [2] [3] [4] [5] have interesting applications to quite a large class of physical phenomena, among them the Kondo problem [6], absorption of polymers on a surface [7] [8] and transport properties of Luttinger liquids [9]. In addition to extensive quantities which can be computed by means of the Bethe Ansatz [10] [11], it is also interesting to analyse the behaviour of local observables in the presence of boundary conditions. In this paper we discuss a simple example of QFT with boundary conditions which has the advantage of being suitable for an exact analysis. The example we consider is the computation of one-point functions of the local operators of the Ising model defined in an half-space. Original lattice derivation of these quantities may be found in the refs. [12] [13] [14]. Before proceeding in our computation, let us briefly recall some general aspects of the problem.

The best way to compute correlation functions of a QFT with boundary is to take full advantage of the solution of the theory in the bulk and of the knowledge of the boundary state [4] [15]. Namely, instead of considering the boundary placed at the spatial coordinate $x=0$, it is most convenient to consider the boundary placed at $t=0$ and described by the boundary state $\mid B>($ Figure 1$)$.
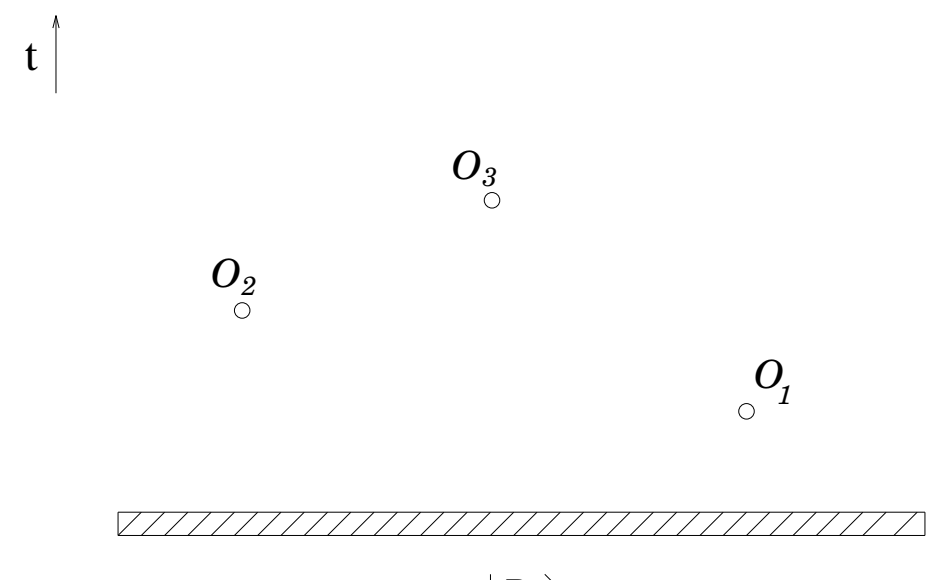

B >

Figure 1. Boundary geometry for the computation of correlators. 
Then, the correlation functions can be expressed as

$$
<\mathcal{O}_{1}\left(x_{1}, t_{1}\right) \ldots \mathcal{O}_{n}\left(x_{n}, t_{n}\right)>=\frac{<0\left|T_{t}\left[\mathcal{O}_{1}\left(x_{1}, t_{1}\right) \ldots \mathcal{O}_{n}\left(x_{n}, t_{n}\right)\right]\right| B>}{<0 \mid B>} .
$$

In this geometry, the Hilbert space of the theory is as in the bulk and therefore, even in the presence of the boundary line, the local operators $\mathcal{O}_{i}$ can be completely characterized by the bulk Form Factors $<\beta_{1}, \ldots, \beta_{n}\left|\mathcal{O}_{i}\right| \beta_{n+1}, \ldots, \beta_{m}>$, where $\beta_{i}$ are the rapidity variables, related to the two-dimensional momenta by $p_{i}^{(0)}=m_{i} \cosh \beta_{i}, p_{i}^{(1)}=m_{i} \sinh \beta_{i}$.

Let us focalize our analysis to the Ising model with a boundary in the low temperature phase $\left(T<T_{c}\right)$ 近. The system can be described in terms of massive fermionic operators $A(\beta)$ and $A^{\dagger}(\beta)$ with the usual anti-commutation relations. The mass $m$ is a linear measurement of the deviation of the temperature with respect to the critical one, $m=2 \pi\left(T_{c}-T\right)$. The spins on the boundary can be subjected to three possible boundary conditions, namely (a) they can be frozen to one of the fixed values \pm 1 ("fixed boundary condition"), (b) they can be completely free to fluctuate ("free boundary condition") or (c) they can be coupled to a boundary magnetic field $h$ ("magnetic boundary condition") The detailed analysis of these three possibilities is given in the original reference [4, and here we simply recall the results for the reflection $S$-matrix $R(\beta)$ associated to each of them. For the free boundary condition we have $R_{+}(\beta)=i \operatorname{coth}\left(\frac{\beta}{2}-\frac{i \pi}{4}\right)$, for the fixed boundary condition $R_{-}(\beta)=-i \tanh \left(\frac{\beta}{2}-\frac{i \pi}{4}\right)$, and finally for the magnetic boundary condition

$$
R_{h}(\beta)=-i \tanh \left(\frac{\beta}{2}-\frac{i \pi}{4}\right) \frac{\kappa-i \sinh \beta}{\kappa+i \sinh \beta},
$$

where $\kappa=1-\frac{h^{2}}{2 m}$. Varying $h$, we can interpolate between the free boundary condition $(h=0)$ and the fixed one $(h \rightarrow \infty)$.

Given an amplitude $R(\beta)$, the corresponding boundary state can be then expressed ast

$$
\text { | } B>=\exp \left[\int_{0}^{\infty} \frac{d \beta}{2 \pi} \widehat{R}(\beta) A^{\dagger}(-\beta) A^{\dagger}(\beta)\right] \mid 0>,
$$

where $\widehat{R}(\beta)=R\left(\frac{i \pi}{2}-\beta\right)$. The structure of the boundary state $|B\rangle$ is such that the states entering its definition consist of pairs of particles with opposite rapidity (Cooper pairs).

1 In order to simplify the notation, from now on we denote the free b.c. with the index $(+)$, the fixed b.c. with the index $(-)$ and the magnetic b.c. with $(h)$.

2 The most general expression of the boundary state may also involve an additional term relative to the zero mode. Here we discard it since it does not enter our following computations. 
We can now use the Form Factors of the Ising model determined in [16] [17] and the expression of the boundary state (1.3) to compute the correlation functions in the presence of the boundary.

\section{One-point and two-point functions of the energy operator}

The simplest correlation function is the one-point function of the energy operator $\epsilon(x, t)$ which can be computed through the formula

$$
\epsilon_{0}(t)=\sum_{n=0}^{\infty}<0|\epsilon(x, t)| n><n \mid B>.
$$

The energy operator couples the vacuum only to the two particle state and for its matrix element in the euclidean space we have

$$
\begin{aligned}
<0|\epsilon(x, t)| \beta_{1}, \beta_{2}>=- & 2 \pi m i \sinh \frac{\beta_{1}-\beta_{2}}{2} \\
& \times \exp \left[-m t\left(\cosh \beta_{1}+\cosh \beta_{2}\right)+i m x\left(\sinh \beta_{1}+\sinh \beta_{2}\right)\right] .
\end{aligned}
$$

Hence the sum (2.1) consists of only one term and the one-point function of the energy operator can be expressed as

$$
\epsilon_{0}(t)=-i m \int_{0}^{\infty} d \beta \sinh \beta \widehat{R}(\beta) e^{-2 m t \cosh \beta} .
$$

Its graphical representation is given in Figure 2.

The one-point function does not depend on $x$, as a consequence of the translation invariance along this axis. The above integral reduces to closed expressions in terms of the modified Bessel functions in the case of free and fixed boundary conditions. Those are given respectively by

$$
\epsilon_{0}^{( \pm)}(t)=\mp m\left[K_{1}(2 m t) \pm K_{0}(2 m t)\right]
$$

In the short distance limit $m t \rightarrow 0$, they have the scaling form $\epsilon_{0}^{( \pm)}(t) \sim \mp \frac{1}{2 t}$, in agreement with the Conformal Field Theory prediction [18]. In the large distance limit, they instead decay exponentially with an extra power term, which is different in the two cases, i.e.

$$
\begin{aligned}
& \epsilon_{0}^{(+)}(t) \sim-m \sqrt{\frac{\pi}{m t}} e^{-2 m t}, \\
& \epsilon_{0}^{(-)}(t) \sim \frac{m}{8} \sqrt{\frac{\pi}{(m t)^{3}}} e^{-2 m t} .
\end{aligned}
$$




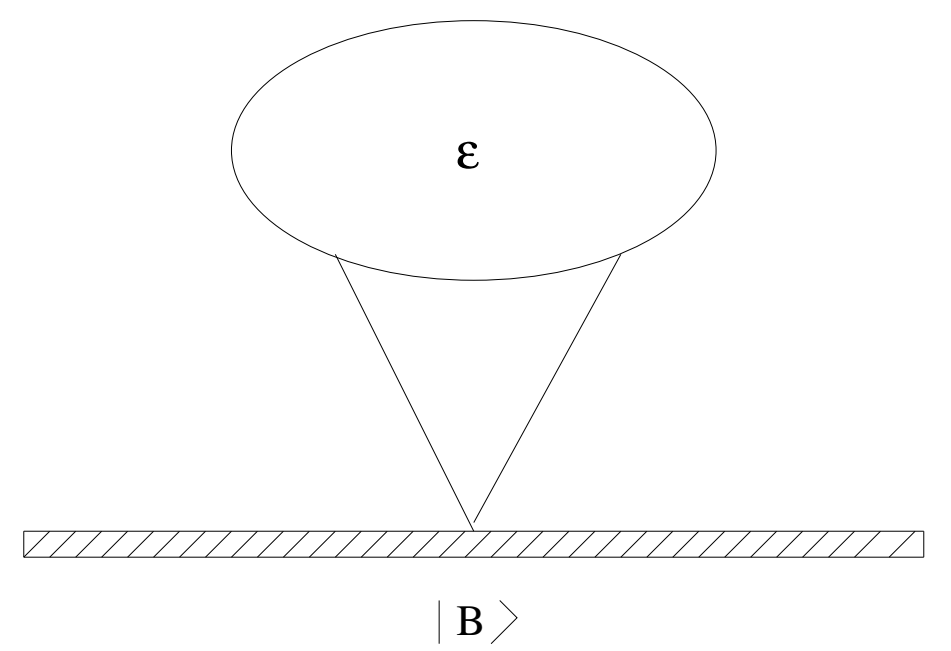

Figure 2. One-point function of the energy operator.

Let us turn our attention to the one-point function of the energy operator in the presence of a boundary magnetic field. This is given by

$$
\epsilon_{0}(t, h)=-m \int_{0}^{\infty} d \beta(\cosh \beta-1) \frac{\cosh \beta+\kappa}{\cosh \beta-\kappa} e^{-2 m t \cosh \beta} .
$$

By varying $h$, we may switch between the free and the fixed boundary conditions, realizing therefore a Renormalization Group flow on the boundary. At first sight, however, it seems impossible to interpolate between the two functions $\epsilon_{0}^{( \pm)}(t)$, which present quite a different behaviour. (Notice, in particular, that the function $\epsilon_{0}^{(-)}(t)$ diverges as $+\infty$ at $t \rightarrow 0$ whereas the other correlation function $\epsilon_{0}^{(+)}(t)$ goes to $-\infty$ near the origin.) To solve this apparent paradox, we have to correctly take into account the interplay between the two variables $t$ and $h$. To this aim, it is convenient to write down a differential equation satisfied by $\epsilon_{0}(t, h)$. Observe that eq. (2.6) can be initially expressed as

$$
\epsilon_{0}(r, h)=-m \int_{0}^{\infty} d \beta \int_{0}^{\infty} d \alpha(\cosh \beta-1)(\cosh \beta+\kappa) e^{-r \cosh \beta-\alpha(\cosh \beta-\kappa)}
$$

$(r \equiv 2 m t)$ and the resulting integral can be expressed in terms of modified Bessel function as

$$
\epsilon_{0}(r, h)=-m e^{-\kappa r} \int_{r}^{\infty} d \eta e^{\kappa \eta}\left[\frac{K_{1}(\eta)}{\eta}+(1-\kappa)\left(K_{0}(\eta)-K_{1}(\eta)\right]\right.
$$


Taking the derivative with respect to $r$ in both sides of this equation, we obtain the following differential equation satisfied by $\epsilon_{0}(r, h)$

$$
\frac{\partial \epsilon_{0}(r, h)}{\partial r}+\kappa \epsilon_{0}(r, h)=m\left[\frac{K_{1}(r)}{r}+(1-\kappa)\left(K_{0}(r)-K_{1}(r)\right)\right]
$$

For $h=0$, using the differential equation satisfied by the Bessel functions, we recover for $\epsilon_{0}(r, h=0)$ the previous result $\epsilon_{0}^{(+)}(t)$, whereas $\epsilon_{0}^{(-)}(t)$ is obtained by taking directly the limit $\kappa \rightarrow-\infty$ in the differential equation.

The differential equation is quite useful to analysing the short and large distance behaviour of the one-point function $\epsilon_{0}(r, h)$. For $r \rightarrow 0$, we can parameterize the solution as $\epsilon_{0}(r, h) \sim C r^{-\delta}$. Substituting into (2.9), it is easy to see that for any finite $\kappa$, the term which determines the short distance behaviour in the RHS is $m \frac{K_{1}(r)}{r}$, therefore $C=-m$ and $\delta=1$, independent of the value of the magnetic field. Hence, as far as $h$ is finite, all the curves $\epsilon_{0}(r, h)$ follow at a short distance scale the behaviour associated to the free boundary condition.

For $r \rightarrow \infty$, we look for a solution of the form $\epsilon_{0}(r, h) \sim e^{-r} r^{-\omega} \sum_{k=0}^{\infty} a_{k} r^{-k}$. Substituting into (2.9), using the large distance expansion of the Bessel functions, and comparing the power series we have then the following cases:

(i) for $\kappa=1, a_{0}=-2 m \sqrt{\frac{\pi}{2}}$ and $\omega=\frac{1}{2}$, so that $\epsilon_{0}(r, h=0) \sim a_{0} e^{-r} r^{-1 / 2}$.

(ii) for $-1<\kappa<1$, we have $a_{0}=-m \sqrt{\frac{\pi}{2}} \frac{1+\kappa}{1-\kappa}$ and $\omega=\frac{3}{2}$, so that $\epsilon_{0}^{(h)}(r) \sim$ $a_{0} e^{-r} r^{-3 / 2}$. In this range $a_{0}<0$ and the function approaches the real axis from below.

(iii) for $\kappa=-1$ the first leading term of the expansion vanishes and for the next leading term we have $a_{1}=-m \frac{27}{16} \sqrt{\frac{\pi}{2}}, \omega=\frac{3}{2}$ and the corresponding solution goes to zero much faster, $\epsilon_{0}(r, h=2 \sqrt{m}) \sim a_{1} e^{-r} r^{-5 / 2}$.

(iv) for $\kappa<-1$, we have $a_{0}=-m \sqrt{\frac{\pi}{2}} \frac{1+\kappa}{1-\kappa}, \omega=\frac{3}{2}$ and $\epsilon_{0}^{(h)} \sim a_{0} e^{-r} r^{-3 / 2}$. In this range $a_{0}>0$ and the function approaches the real axis from above.

The one-point functions relative to some of the above cases are shown in Figure 3.

The result of this analysis is that for the boundary magnetic field smaller than the critical value $h_{c}=2 \sqrt{m}$, the one-point function does not have any zero at finite value of $r$ whereas, for $h>h_{c}$, it crosses the horizontal axes at a finite value of $r$, and its large $r$ behaviour closely follows the behaviour relative to fixed boundary condition.

The above picture is consistent with a Renormalization Group analysis. In fact, the boundary magnetic field is a relevant operator which therefore cannot affect the behaviour 


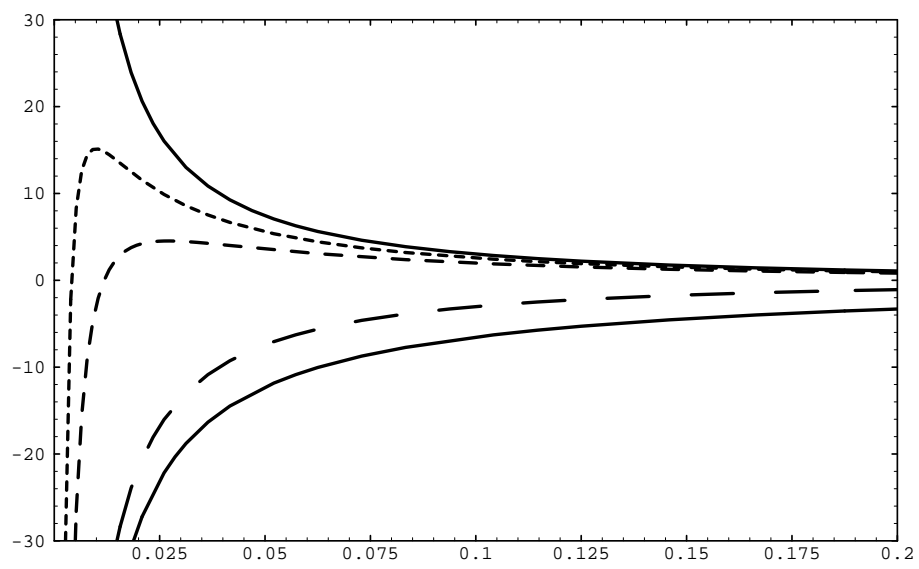

Figure 3. One-point function of the energy operator versus $(m t)$ for different values of the boundary magnetic field. Upper full line: fixed b.c.. Lower full line: free b.c.. Long dashed line: $h<h_{c}$. Short dashed lines: curves with $h>h_{c}$.

of the correlation functions near the boundary, as far as it assumes finite values. Hence, sufficiently close to $r=0$ the boundary always appears subject to free boundary condition. The boundary magnetic field, however, influences the observables at large distance scales and when $h>h_{c}$, the boundary always appears as subject to the fixed boundary condition for an observer placed at $r \rightarrow \infty$. In an intermediate scale, there is a non-trivial crossover between the two different behaviours, which becomes rather sharp with increasing $h$. The function $r(h)$, implicitly defined as the zeros of the one-point function $\epsilon_{0}(r(h), h)=0$, 


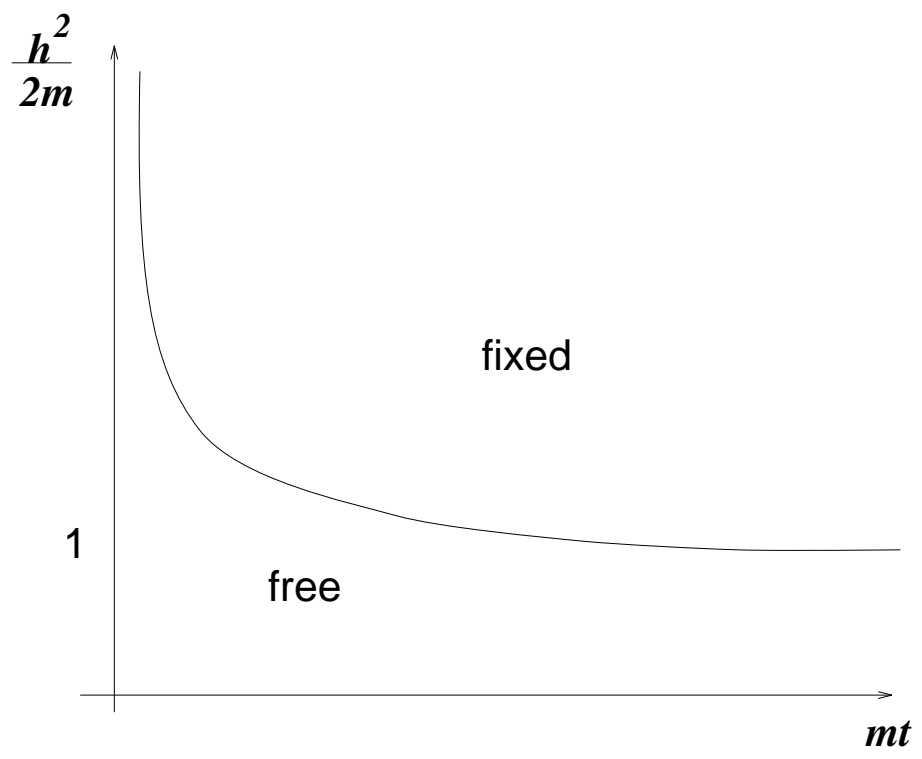

Figure 4. Zeros of the one-point function $\epsilon_{0}(r(h), h)$.

may be interpreted as a phase diagram of the theory which divides the free and the fixed boundary condition regions (Fig. 4).

It is interesting to study the massless limit of the one-point function (2.6). This corresponds to the physical situation of a critical Ising model in the bulk but in the presence of a boundary condition which breaks the conformal invariance of the model. With the change of variable $y=2 m t \cosh \beta$, the integral (2.6) becomes

$$
\epsilon_{0}(t, h)=\frac{1}{2 t} \int_{2 m t}^{\infty} d y e^{-t} \sqrt{\frac{y-2 m t}{y+2 m t}} \frac{y+2 m t \kappa}{y-2 m t \kappa}
$$

In the limit $m \rightarrow 0$, we have $2 m t \kappa \rightarrow-h^{2} t \equiv-z$ and the one-point function of the energy operator can be expressed as

$$
\epsilon_{0}(t, h)=\frac{1}{2 t}\left[1+2 z e^{z} \operatorname{Ei}(-z)\right]
$$

where $\operatorname{Ei}(z)$ is the exponential integral function.

Let us consider now the connected two-point function of the energy operator in presence of boundary magnetic field,

$$
G_{c}\left(x_{1}, t_{1} ; x_{2}, t_{2} ; h\right)=<\epsilon\left(x_{1}, t_{1}\right) \epsilon\left(x_{2}, t_{2}\right)>-\epsilon_{0}\left(t_{1}, h\right) \epsilon_{0}\left(t_{2}, h\right)
$$


By translation invariance along the $x$ axis, this function depends on the difference $x=$ $x_{2}-x_{1}$. On the other hand, the presence of the boundary in the time direction implies a dependence of $G$ on the two variables $t_{1}$ and $t_{2}$. It is convenient to define $t \equiv t_{2}-t_{1}$, $\bar{t} \equiv=t_{2}+t_{1}$ and $r \equiv \sqrt{x^{2}+t^{2}}$. The correlation function (2.12) can be easily computed by using eqs. (1.1) and the form factor (2.2) . Its closed form is given by

$$
\begin{gathered}
G_{c}\left(x_{1}, t_{1} ; x_{2}, t_{2} ; h\right)=m^{2}\left[\left(\frac{\partial}{\partial(m x)} K_{0}(m r)+F(m x, m \bar{t})\right)^{2}+\left(\frac{\partial}{\partial(m t)} K_{0}(m r)\right)^{2}\right. \\
\left.-\left(\frac{\partial}{\partial(m \bar{t})} F(m x, m \bar{t})\right)^{2}-\left(K_{0}(m r)+\frac{\partial}{\partial(m x)} F(m x, m \bar{t})\right)^{2}\right]
\end{gathered}
$$

where we have introduced the function

$$
F(m \rho, m \tau) \equiv-\frac{i}{2} \int_{-\infty}^{+\infty} d q \frac{\cosh q+\kappa}{\cosh q-\kappa} \tanh \frac{q}{2} \exp (-m \tau \cosh q+i m \rho \sinh q) .
$$

In the massless limit, the two-point function can be expressed as

$$
G_{c}\left(x_{1}, t_{1} ; x_{2}, t_{2} ; h\right)=\frac{1}{r^{2}}+\mathcal{F}(x, \bar{t} ; h) \mathcal{F}(-x, \bar{t} ; h)
$$

where

$$
\mathcal{F}(\rho, \tau ; h)=\int_{0}^{\infty} d q \frac{2 q-h^{2}}{2 q+h^{2}} \exp (-\tau q+i \rho q) .
$$

It is easy to see that for free and fixed boundary conditions, eq. 2.15) correctly reduces to the expression obtained by methods of boundary conformal field theory [2] .

\section{One-point function of the magnetization operator}

Let us consider now the one-point correlation functions of the Ising magnetization field $\sigma(x, t)$. This can be computed by using the formula

$$
\sigma_{0}(t)=\sum_{n=0}^{\infty}\langle 0|\sigma(x, t)| n\rangle\langle n \mid B\rangle,
$$

and by translation invariance, it only depends on $t$. We denote by $\sigma_{0}^{(+)}(t)$ the one-point function relative to the free b.c., by $\sigma_{0}^{(-)}(t)$ the one-point function with fixed b.c., and finally by $\sigma_{0}(t, h)$ the one-point function in the presence of an arbitrary boundary magnetic 
field $h$. In order to compute these one-point functions, we need the bulk Form Factors of the magnetization operator 3 , given by [16] [17:

$$
\left\langle 0|\sigma(0)| \beta_{1} \cdots \beta_{2 n}\right\rangle=i^{n} \prod_{i<j} \tanh \left(\frac{\beta_{i}-\beta_{j}}{2}\right) \text {. }
$$

Since the boundary state $|B\rangle$ consists of pairs of particles with opposite rapidity, we have to specialize the previous formula and evaluate

$$
\begin{aligned}
\left\langle 0|\sigma(0)|-\beta_{1}, \beta_{1}, \cdots-\beta_{n}, \beta_{n}\right\rangle & =(-i)^{n} \prod_{i=1}^{n} \tanh \beta_{i} \prod_{i<j}\left(\tanh \frac{\beta_{i}-\beta_{j}}{2} \tanh \frac{\beta_{i}+\beta_{j}}{2}\right)^{2}= \\
& =(-i)^{n} \prod_{i=1}^{n} \tanh \beta_{i} \prod_{i<j}\left(\frac{\cosh \beta_{i}-\cosh \beta_{j}}{\cosh \beta_{i}+\cosh \beta_{j}}\right)^{2} .
\end{aligned}
$$

Noting that

$$
\prod_{i<j}\left(\frac{\cosh \beta_{i}-\cosh \beta_{j}}{\cosh \beta_{i}+\cosh \beta_{j}}\right)^{2}=\operatorname{det}\left(\frac{2 \sqrt{\cosh \beta_{i} \cosh \beta_{j}}}{\cosh \beta_{i}+\cosh \beta_{j}}\right) \equiv \operatorname{det} W\left(\beta_{i}, \beta_{j}\right)
$$

we can express the one-point function $\sigma_{0}^{( \pm)}(t)$ as a Fredholm determinant, namely,

$$
\begin{aligned}
\sigma_{0}^{( \pm)}(t) & =\sum_{i=0}^{\infty} \frac{1}{n !} \int_{0}^{+\infty} \frac{d \beta_{1}}{2 \pi} \cdots \int_{0}^{+\infty} \frac{d \beta_{n}}{2 \pi}\left(\prod_{k=0}^{n}\left(-i \tanh \beta_{k} \widehat{R}_{ \pm}\left(\beta_{k}\right)\right)\right) \operatorname{det} W\left(\beta_{i}, \beta_{j}\right)= \\
& =\operatorname{Det}\left(1-z_{ \pm} V_{( \pm)}(t)\right)
\end{aligned}
$$

where the kernels are given by

$$
V_{( \pm)}\left(\beta_{i}, \beta_{j}, t\right)=\frac{e^{( \pm)}\left(\beta_{i}, t\right) e^{( \pm)}\left(\beta_{j}, t\right)}{\cosh \beta_{i}+\cosh \beta_{j}}, \quad e^{( \pm)}(\beta, t)=\sqrt{\cosh \beta \pm 1} e^{-m t \cosh \beta}
$$

In the above formula, the physical correlator is obtained for $z_{ \pm}= \pm 1 /(2 \pi)$, but it is convenient to regard the above expressions as functions of the parameters $z_{ \pm}$.

The one-point function $\sigma_{0}(t, h)$ can also be cast into a Fredholm determinant form as

$$
\sigma_{0}(t, h)=\operatorname{Det}(1-z V(t, h))
$$

3 With our normalization of the Form Factors, the conformal limit of the two-point function in the bulk is given by $\langle\sigma(r) \sigma(0)\rangle=\mathcal{F}^{2} r^{-1 / 4}$, where $\mathcal{F}=2^{-1 / 12} e^{1 / 8} A^{-3 / 2} m^{-1 / 8}=$ $0.73642778 m^{-1 / 8}$, with $A=1.282427$ the Glasher constant. 
where the kernel is now given by

$$
\begin{aligned}
V\left(\beta_{i}, \beta_{j}, t, h\right) & =\frac{E\left(\beta_{i}, t, h\right) E\left(\beta_{j}, t, h\right)}{\cosh \beta_{i}+\cosh \beta_{j}}, \\
E(\beta, t, h) & =\sqrt{(\cosh \beta-1) \frac{\cosh \beta+\kappa}{\cosh \beta-\kappa}} e^{-m t \cosh \beta} .
\end{aligned}
$$

In this case, the physical correlator is obtained for $z=1 /(2 \pi)$.

It is quite easy to show that the crossover phenomenon previously discussed for the one-point function of the energy operator also takes place for $\sigma_{0}(t, h)$. Let us initially consider the short distance behaviour of eqs. (3.5) and (3.7). As proved in appendix A, in the limit $m t \rightarrow 0$, we have

$$
\begin{aligned}
& \sigma_{0}^{+}(t) \sim(2 t)^{3 / 8}, \\
& \sigma_{0}^{-}(t) \sim(2 t)^{-1 / 8} .
\end{aligned}
$$

Concerning the behaviour of the one-point function $\sigma_{0}(t, h)$ in the limit $m t \rightarrow 0$, its power law singularity is independent of $h$, as far as $h$ is finite, and coincides with that one relative to the free boundary condition, i.e. $\sigma_{0}(t, h) \sim(2 t)^{3 / 8}$. Hence, varying $h$, all the curves $\sigma_{0}(t, h)$ follow at short distance the power-law behaviour dictated by the free boundary conditions.

Let us evaluate now the large distance behaviour. For the fixed boundary conditions, we have

$$
\sigma_{0}^{-}(t)=\left[1+\frac{1}{16 \sqrt{\pi}} \frac{e^{-2 m t}}{(m t)^{3 / 2}}+\cdots\right],
$$

i.e. this function approaches the bulk expectation value from above. For the free boundary condition, we have instead

$$
\sigma_{0}^{+}(t)=\left[1-\frac{1}{2 \sqrt{\pi}} \frac{e^{-2 m t}}{(m t)^{1 / 2}}+\cdots\right]
$$

i.e. the bulk expectation value is reached from below. In the presence of a boundary magnetic field, the asymptotical approach to the bulk vacuum expectation value is given for $\kappa \neq-1$ by the expression

$$
\sigma_{0}(t, h)=\left[1-\frac{1}{16 \sqrt{\pi}} \frac{1+\kappa}{1-\kappa} \frac{e^{-2 m t}}{(m t)^{3 / 2}}+\cdots\right],
$$

whereas for $\kappa=-1$

$$
\sigma_{0}(t, h)=\left[1-\frac{3}{128 \sqrt{\pi}} \frac{e^{-2 m t}}{(m t)^{5 / 2}}+\cdots\right]
$$




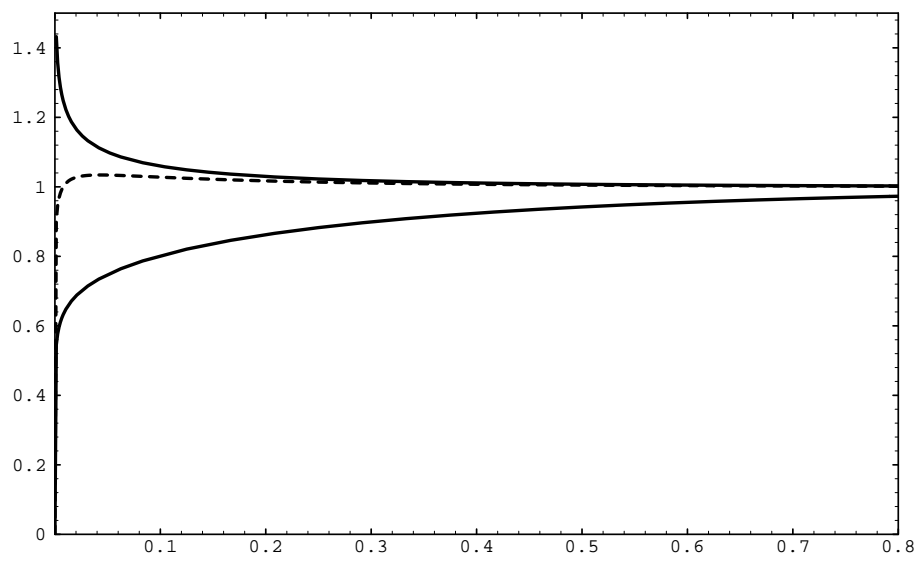

Figure 5. One-point function of the magnetization operator versus $(m t)$. Upper full line: fixed b.c.. Lower full line: free b.c.. Dashed line: curve with $h>h_{c}$.

From (3.12) we see that for $-1<\kappa<1$, the curve reaches its asymptotic behaviour from below, whereas for $\kappa<-1$ from above. At $\kappa=-1$ there is a faster decreasing behaviour. Hence, also for the one-point function of the magnetization operator there is the typical Renormalization Group crossover between free and fixed boundary conditions, moving away from the boundary (Fig. 5).

\section{Differential equation for the magnetization one-point function}

Bariev has shown that for the free and fixed boundary condition, the one-point functions of the magnetization can be expressed in terms of a solution to the Painlevé differ- 
ential equation [14]. He started from a lattice description, from which he obtained the same Fredholm determinant expressions as we directly obtained by using the form factor approach. In this section we derive differential equations for arbitrary magnetic field $h$ using the general techniques developed in [19] [20]. As we will show, these will turn out to be coupled non-linear partial differential equations in the variables $t$ and $\kappa$.

Making the change of variable $u \equiv \cosh \beta$, we can initially write the one-point function $\sigma_{0}(t, h)$ as

$$
\langle 0|\sigma(t)| B(h)\rangle=\operatorname{det}(1+\mathcal{V})=\sum_{n=0}^{\infty} \frac{1}{n !} \int_{1}^{+\infty} \frac{d u_{1}}{2 \pi} \cdots \int_{1}^{+\infty} \frac{d u_{n}}{2 \pi} \operatorname{det} \mathcal{V}\left(u_{i}, u_{j}, t\right)
$$

where

$$
\begin{aligned}
\mathcal{V}\left(u_{i}, u_{j}, t\right) & =\frac{e\left(u_{i}, t\right) e\left(u_{j}, t\right)}{u_{i}+u_{j}}, \\
e(u, t) & =\left(\frac{u-1}{u+1}\right)^{1 / 4} \sqrt{\frac{\kappa+u}{\kappa-u}} e^{-m t u} .
\end{aligned}
$$

One first needs the resolvent $R_{ \pm}$, defined as follows:

$$
\left(1-R_{ \pm}\right)(1 \pm \mathcal{V})=1
$$

In the following, it will always be implied that Fredholm operators are multiplied in the usual way, e.g.

$$
(R V)(u, v)=\int_{1}^{+\infty} \frac{d w}{2 \pi} R(u, w) V(w, v)
$$

and similarly for multiplication of operators with functions:

$$
(R e)(u)=\int_{1}^{+\infty} \frac{d v}{2 \pi} R(u, v) e(v)
$$

Resolvents for the kernels of the type (3.6) were described in [21]. Define functions $f^{ \pm}(u)$ by the formulas

$$
(1 \pm \mathcal{V}) f^{ \pm}=e \quad \Longleftrightarrow \quad f^{ \pm}=\left(1-R_{ \pm}\right) e
$$

Then

$$
R_{ \pm}(u, v)=H(u, v) \pm F(u, v)
$$

where

$$
\begin{aligned}
& H(u, v)=\frac{f^{-}(u) f^{+}(v)-f^{+}(u) f^{-}(v)}{2(u-v)} \\
& F(u, v)=\frac{f^{+}(u) f^{-}(v)+f^{-}(u) f^{+}(v)}{2(u+v)}
\end{aligned}
$$


The next step is to identify variables with the property that derivatives of the kernel $\mathcal{V}$ with respect to such variables acts as a projector, i.e. removes the $1 /(u+v)$ factor. The spacial variable $t$ obviously has this property:

$$
\partial_{t} \mathcal{V}(u, v)=-m e(u) e(v) .
$$

One also has

$$
\partial_{\kappa} \mathcal{V}(u, v)=\left(u v-\kappa^{2}\right) e^{\prime}(u) e^{\prime}(v)
$$

where

$$
e^{\prime}(u)=\frac{e(u)}{u^{2}-\kappa^{2}} .
$$

For future convenience, define also

$$
\widehat{e}(u)=\frac{u}{u^{2}-\kappa^{2}} e(u) .
$$

The rest of the derivation of correlation functions parallels closely the treatment of Ising 2-point functions given in 21] with $z, \bar{z}$ replaced by $r, \kappa$. Let

$$
\tau_{ \pm}=\operatorname{Det}(1 \pm \mathcal{V})
$$

Then

$$
\begin{aligned}
\partial_{t} \log \tau_{ \pm} & =\partial_{t} \operatorname{Tr} \log (1 \pm \mathcal{V})=\operatorname{Tr}\left(1-R_{ \pm}\right) \partial_{t} \mathcal{V} \\
& =\mp m P_{ \pm}
\end{aligned}
$$

where

$$
P^{ \pm}=<e, f^{ \pm}>\equiv \int \frac{d u}{2 \pi} e(u) f^{ \pm}(u) .
$$

Next, we compute $\partial_{\kappa} P_{ \pm}$, for which we need $\partial_{\kappa} f^{ \pm}$. Taking the derivative of (4.6), one obtains

$$
(1 \pm \mathcal{V}) \partial_{\kappa} f^{ \pm} \pm \partial_{\kappa} \mathcal{V} f^{ \pm}=\widehat{e}
$$

The latter implies

$$
(1 \pm \mathcal{V}) \partial_{\kappa} f^{ \pm} \mp \kappa^{2} e^{\prime}<e^{\prime}, f^{ \pm}> \pm \widehat{e}<\widehat{e}, f^{ \pm}>=\widehat{e}
$$

Define the additional functions

$$
\widehat{f}^{ \pm}=\left(1-R_{ \pm}\right) \widehat{e}, \quad f^{\prime \pm}=\left(1-R_{ \pm}\right) e^{\prime} .
$$


Then multiplying (4.17) by $\left(1-R_{ \pm}\right)$, one obtains

$$
\partial_{\kappa} f^{ \pm}=\widehat{f}^{ \pm} \pm \kappa^{2} D^{ \pm} f^{\prime \pm} \mp \widehat{D}^{ \pm} \widehat{f}^{ \pm}
$$

where

$$
\begin{aligned}
& D^{ \pm}=<e^{\prime}, f^{ \pm}>=<e, f^{\prime \pm}>, \\
& \widehat{D}^{ \pm}=<\widehat{e}, f^{ \pm}>=<e, \widehat{f}^{ \pm}>.
\end{aligned}
$$

One then finds

$$
\begin{aligned}
\partial_{\kappa} P^{ \pm} & =<\partial_{\kappa} e, f^{ \pm}>+<e, \partial_{\kappa} f^{ \pm}> \\
& =2 \widehat{D}^{ \pm} \pm \kappa^{2}\left(D^{ \pm}\right)^{2} \mp\left(\widehat{D}^{ \pm}\right)^{2}
\end{aligned}
$$

We can also obtain expressions for $\partial_{t} D^{ \pm}$and $\partial_{t} \widehat{D}^{ \pm}$. For this, one needs $\partial_{t} f^{ \pm}$. This is the same as in [21]:

$$
\partial_{t} f^{ \pm}=m\left[ \pm\left(P^{+}+P^{-}\right) f^{ \pm}-u f^{\mp}\right]
$$

One then has

$$
\begin{aligned}
\partial_{t} D^{ \pm} & =<\partial_{t} e^{\prime}, f^{ \pm}>+<e^{\prime}, \partial_{t} f^{ \pm}> \\
& =m\left[ \pm\left(P^{+}+P^{-}\right) D^{ \pm}-\widehat{D}^{+}-\widehat{D}^{-}\right]
\end{aligned}
$$

Similarly,

$$
\partial_{t} \widehat{D}^{ \pm}=m\left[ \pm\left(P^{+}+P^{-}\right) \widehat{D}^{ \pm}-\left(P^{+}+P^{-}\right)-\kappa^{2}\left(D^{+}+D^{-}\right)\right]
$$

Finally, there is one non-trivial constraint among the $D, \widehat{D}$ potentials, which is proven in the appendix B:

$$
\widehat{D}^{+}=\widehat{D}^{-}-\widehat{D}^{+} \widehat{D}^{-}+\kappa^{2} D^{+} D^{-}
$$

We can summarize the above results as follows. Define

$$
\begin{array}{ll}
P=P^{+}+P^{-}, & Q=P^{+}-P^{-}, \\
C=2+\widehat{D}^{-}-\widehat{D}^{+}, & \widetilde{C}=-\left(\widehat{D}^{+}+\widehat{D}^{-}\right), \\
D=D^{+}+D^{-}, & \widetilde{D}=D^{+}-D^{-} .
\end{array}
$$

Then,

$$
\begin{aligned}
& \partial_{t} \log \left(\tau_{+} / \tau_{-}\right)=-m P \\
& \partial_{t} \log \left(\tau_{+} \tau_{-}\right)=-m Q \\
& \partial_{\kappa} P=\kappa^{2} D \widetilde{D}-C \widetilde{C} \\
& \partial_{\kappa} Q=2-\frac{1}{2}\left(C^{2}+\widetilde{C}^{2}\right)+\frac{\kappa^{2}}{2}\left(D^{2}+\widetilde{D}^{2}\right)
\end{aligned}
$$


and

$$
\begin{aligned}
& \partial_{t} D=m[2 \widetilde{C}+P \widetilde{D}], \\
& \partial_{t} \widetilde{D}=m P D, \\
& \partial_{t} C=m P \widetilde{C}, \\
& \partial_{t} \widetilde{C}=m\left[P C+2 \kappa^{2} D\right] .
\end{aligned}
$$

The constraint reads

$$
C^{2}-\widetilde{C}^{2}+\kappa^{2}\left(D^{2}-\widetilde{D}^{2}\right)=4
$$

The equations (4.27) indicate that $\partial_{t} \partial_{\kappa} \log \left(\tau_{ \pm}\right)$can be expressed in terms of the four functions $C, \widetilde{C}, D, \widetilde{D}$. In turn, the function $P$ can be eliminated from (4.28) to obtain

differential equations involving $C, \widetilde{C}, D, \widetilde{D}$ only. For instance, expressing $P=\partial_{t} \widetilde{D} / m D$, and using the expression for $\partial_{\kappa} P$, one finds:

$$
D \partial_{\kappa} \partial_{t} \widetilde{D}-\partial_{t} \widetilde{D} \partial_{\kappa} D=m D^{2}\left(\kappa^{2} D \widetilde{D}-C \widetilde{C}\right)
$$

Three additional differential equations of the same kind can be similarly obtained by eliminating $P$ from each of the equations in (4.28).

\section{Concluding Remarks}

In this paper we have studied the effects induced by a boundary Renormalization Group flow on the correlation functions of the two-dimensional Ising model. Using the form factors of the local energy and magnetization operators, we have computed their onepoint functions in the presence of a boundary magnetic field as sums on the intermediate states of the bulk theory. The integral representations of the correlation functions can be used to derive the differential equations which they satisfy. In view of the "free" nature of the energy operator in the Ising model, its one-point functions in the presence of a boundary magnetic field is a solution of a linear non-homogeneous differential equation, given in (2.9). On the contrary, the magnetization operator is an interacting field and for its one-point function in the presence of a boundary magnetic field, we obtain the system of non-linear differential equations (4.27), (4.28), with the constraint expressed by (4.29). The rather different mathematical structure of these differential equations reflects the distinct dynamics of those fields.

Some simplications in the equations are expected in the massless limit of the theory. This corresponds to the Ising model with an infinite correlation length in the bulk but 
with a violation of the conformal symmetry induced by the boundary interaction. We have analysed this interesting situation for the cases of the one-point and the two-point functions of the energy operator. Concerning the one-point function of the magnetization operator, it is known that it satisfies in the massless limit a linear differential equation of hypergeometric type [22]. It would be interesting to obtain directly the result of ref. [22] by analysing the massless limit of the system of non-linear differential equations derived in this paper.

\section{Acknowledgements}

We would like to thank V. Korepin for useful discussions. This work is supported by an Alfred P. Sloan Foundation fellowship, and the National Science Foundation in part through the National Young Investigator program.

\section{Appendix A.}

In this appendix we calculate the power law singularity of the one-point function of the magnetization operator. Let us start by analysing the free and fixed boundary conditions. An equivalent expression for $\sigma_{0}^{( \pm)}(t)$ is given by

$$
\begin{aligned}
\sigma_{0}^{( \pm)}(t) & =\operatorname{det}\left(1-z_{ \pm} V_{( \pm)}\right)=\exp \left[\operatorname{Tr} \log \left(1-z_{ \pm} V_{( \pm)}\right)\right]= \\
& =\exp \left[-\sum_{n=1}^{\infty} \frac{z_{ \pm}^{n}}{n} \int_{0}^{+\infty} d \beta_{1} \cdots \int_{0}^{+\infty} d \beta_{n} \prod_{i=1}^{n} e^{-2 m t \cosh \beta_{i}} \frac{\cosh \beta_{i} \pm 1}{\cosh \beta_{i}+\cosh \beta_{i+1}}\right]= \\
& \equiv \exp \left[-\sum_{n=1}^{\infty} \frac{z_{ \pm}^{n}}{n} Z_{ \pm}^{(n)}(t)\right]
\end{aligned}
$$

(where $\beta_{n+1} \equiv \beta_{1}$ ). Using now

$$
\frac{1}{\cosh \beta_{i}+\cosh \beta_{i+1}}=\int_{0}^{+\infty} d \alpha e^{-\alpha\left(\cosh \beta_{i}+\cosh \beta_{i+1}\right)}
$$

the coefficients $Z_{ \pm}^{(n)}(t)$ in A.1 can be expressed as

$$
\begin{aligned}
Z_{ \pm}^{(n)}(t) & =\int_{m t}^{+\infty} d \alpha_{1} \cdots \int_{m t}^{+\infty} d \alpha_{n}\left[K_{1}\left(\alpha_{1}+\alpha_{2}\right) \pm K_{0}\left(\alpha_{1}+\alpha_{2}\right)\right] \\
& \times\left[K_{1}\left(\alpha_{2}+\alpha_{3}\right) \pm K_{0}\left(\alpha_{2}+\alpha_{3}\right)\right] \cdots\left[K_{1}\left(\alpha_{n}+\alpha_{1}\right) \pm K_{0}\left(\alpha_{n}+\alpha_{1}\right)\right]
\end{aligned}
$$


where $K_{1}(x)$ and $K_{0}(x)$ are modified Bessel functions. For $m t \rightarrow 0$, the leading singularity of these expressions comes from

$$
Z_{ \pm}^{(n)}(t) \sim \int_{m t}^{+\infty} d \alpha_{1} \cdots \int_{m t}^{+\infty} d \alpha_{n} K_{1}\left(\alpha_{1}+\alpha_{2}\right) K_{1}\left(\alpha_{2}+\alpha_{3}\right) \cdots K_{1}\left(\alpha_{n}+\alpha_{1}\right)
$$

It is easy to estimate the sum of the most singular terms. To do this, let us express $\sigma_{0}^{( \pm)}(t)$ in terms of the eigenvalues $\lambda_{ \pm}(t)$ of the integral operators $V_{ \pm}$and their multiplicity $a_{i}(t)$ as

$$
\sigma_{0}^{( \pm)}(t)=\prod_{i=1}^{\infty}\left(1-z_{ \pm} \lambda_{ \pm}^{(i)}(t)\right)^{a_{i}(t)}
$$

As far as $(m t)$ is finite, the kernel is square integrable. However, when $(m t) \rightarrow 0$, the operator becomes unbounded. In this limit, the eigenvalues becomes dense in the interval $(0, \infty)$ according to the distribution

$$
\lambda(p)=\frac{2 \pi}{\cosh \pi p}
$$

whereas, from Mercer's theorem, their multiplicity grows logarithmically as $a_{i} \sim \frac{1}{\pi} \ln \frac{1}{m x}$. Therefore

$$
\log \sigma_{0}^{( \pm)}(t)=x_{ \pm} \log t
$$

where the critical exponents $x_{ \pm}$of the magnetization operator relative to free and fixed boundary conditions are given by

$$
x_{ \pm}=\frac{1}{\pi^{2}} \int_{0}^{\infty} d p \ln \left(1-\frac{2 \pi z_{ \pm}}{\cosh p}\right)=\frac{1}{8}-\frac{1}{2 \pi^{2}} \arccos ^{2}\left(-2 \pi z_{ \pm}\right)
$$

Substituting the values of $z_{ \pm}$we obtain $x=3 / 8$ for the free b.c. and $x=-1 / 8$ for the fixed b.c.

Repeating the same analysis for the one-point function in the presence of the boundary magnetic field $h$ by using the formula (A.1), it is easy to see that, as far as $h$ is finite, the most leading singularity of the corresponding $Z^{(n)}$ is always given by (A.4) and can be estimated according to the equation (A.8) with $z=1 /(2 \pi)$, i.e. $x=3 / 8$. 


\section{Appendix B.}

Here, we relate the functions $\widehat{f}^{ \pm}, f^{\prime \pm}$ to the functions $f^{ \pm}$and prove the constraint (4.25).

Using the identity

$$
\frac{1}{u+v} \frac{1}{v+k}=\frac{1}{u-k}\left(\frac{1}{v+k}-\frac{1}{u+v}\right),
$$

one first easily shows that

$$
\begin{aligned}
& H(u, v) \frac{1}{v+k}=\frac{1}{u+k} H(u, v)+\frac{1}{2} \frac{1}{u+k} \frac{1}{v+k}\left(f^{-}(u) f^{+}(v)-f^{+}(u) f^{-}(v)\right), \\
& F(u, v) \frac{1}{v+k}=-\frac{1}{u-k} F(u, v)+\frac{1}{2} \frac{1}{u-k} \frac{1}{v+k}\left(f^{+}(u) f^{-}(v)+f^{-}(u) f^{+}(v)\right) .
\end{aligned}
$$

From the definition,

$$
f^{\prime \pm}(u)=\left(\left(1-R_{ \pm}\right) \frac{e(v)}{v^{2}-\kappa^{2}}\right)(u)
$$

Factorizing $v^{2}-\kappa^{2}=(v+\kappa)(v-\kappa)$ in the above equation, and applying (B.2) twice, one obtains

$$
f^{\prime \pm}(u)=\frac{1}{u^{2}-\kappa^{2}}\left[\left(1 \pm Q^{\mp}\right) f^{ \pm} \mp \kappa D^{\mp} f^{ \pm} \mp u f^{\mp} D^{ \pm}\right]
$$

where

$$
Q^{ \pm}=<f^{ \pm}, \frac{e(u)}{u-\kappa}>=\widehat{D}^{ \pm}+\kappa D^{ \pm}
$$

Using the identities

$$
\begin{aligned}
& H(u, v) v=u H(u, v)+\frac{1}{2}\left(f^{+}(u) f^{-}(v)-f^{-}(u) f^{+}(v)\right), \\
& F(u, v) v=-u F(u, v)+\frac{1}{2}\left(f^{+}(u) f^{-}(v)+f^{-}(u) f^{+}(v)\right)
\end{aligned}
$$

one also finds

$$
\widehat{f}^{ \pm}(u)=u f^{\prime \mp} \mp D^{\mp} f^{ \pm}
$$

The constraint (4.25) follows from using (B.7), (B.4) to express $\widehat{f}^{ \pm}$in terms of $f^{ \pm}$in the equation $\widehat{D}^{ \pm}=<\widehat{e}, f^{ \pm}>=<e, \widehat{f}^{ \pm}>$. 


\section{References}

[1] K. Binder, in Phase transitions and critical phenomena, vol. 8, ed. C. Domb and J. Lebowitz (Academic Press, London 1983).

[2] J.L. Cardy, Nucl. Phys. B 240 [FS12] (1984), 514; Nucl. Phys. B 324 (1989), 581.

[3] I. Cherednik, Theor. Math. Phys. 61 (1984), 997.

[4] S. Ghoshal and A. Zamolodchikov, Int. Journ. Mod. Phys. A 9 (1994), 3841.

[5] A. Fring and R. Koberle, Nucl. Phys. B 419 (1994), 647; Nucl. Phys. B 421 (1994), 159.

[6] I. Affleck and A. Ludwig, Nucl. Phys. B 360 (1991), 641; Phys. Rev. B 48 (1993), 7297.

[7] E. Eisenriegler, Polymers Near Surfaces (World Scientific Publishing, 1993).

[8] P. Fendley and H. Saleur, J. Phys. A (1994), L789.

[9] P. Fendley, A. Ludwig and H. Saleur, Exact non-equilibrium transport through point contacts in quantum wires and fractional quantum Hall effect, preprint UCS-95-007.

[10] R. Chatterjeee, Mod. Phys. Lett. A 10 (1995), 937.

[11] A. LeClair, G. Mussardo, H. Saleur and S. Skorik, Boundary energy and boundary states in integrable quantum field theories, hep-th/9503227, to appear on Nucl. Phys. B.

[12] B.M. McCoy and T.T. Wu, Phys. Rev. 162 (1967) 436; Phys. Rev. 174 (1968) 546.

[13] B.M. McCoy and T.T. Wu, The Two Dimensional Ising Model, Harvard University Press, Cambridge 1973.

[14] R.Z. Bariev, Teor. Mat. Fiz. 40 (1979), 40; Teor. Mat. Fiz. 42 (1980), 262; Teor. Mat. Fiz. 77 (1988), 1090.

[15] G. Delfino, G. Mussardo and P. Simonetti, Nucl. Phys. B 432 [FS] (1994), 518.

[16] B. Berg, M. Karowski and P. Weisz, Phys. Rev. D 19 (1979), 2477.

[17] V.P. Yurov and Al.B. Zamolodchikov, Int. J. Mod. Phys. A 6 (1991), 3419; J.L. Cardy and G. Mussardo, Nucl. Phys. B 340 (1990), 387.

[18] J.L Cardy and D.C. Lewellen, Phys. Lett. B 259 (1991), 274.

[19] A. R. Its, A. G. Izergin, V. E. Korepin, and N. A. Slavnov, Int. J. Mod. Phys. B4 (1990) 1003.

[20] V. E. Korepin, N. M. Bogoliubov and A. G. Izergin, Quantum Inverse Scattering Method and Correlation Functions, (Cambridge Univ. Press, Cambridge, 1993).

[21] D. Bernard and A. LeClair, Nucl. Phys. B426 [FS] (1994) 534.

[22] R. Chatterjee and A. Zamolodchikov, Local Magnetization in Critical Ising Model with Boundary Magnetic Field, hep-th/9311165. 